impulsivity not influenced by external stimuli, is the main hallmark of the excited variety of catatonia. ${ }^{[1]}$ The finding of one catatonic sign should raise the suspicion of this condicion and a full clinical assessment should be undertaken, preferably using a standardized rating scale such as the Bush-Francis Catatonia Rating Scale that also measures the severity of the signs. ${ }^{[2]}$ The use of such scales improves the detection of this syndrome. ${ }^{[2]}$ According to the DSM 5, to make the diagnosis of catatonia is needed the presence of 3 or more signs of catatonia of the 12 above-described. ${ }^{[3]}$

As we mentioned, catatonia has been observed in a wide variety of general medical, neurological and psychiatric disorders as well as associated with drugs and toxic substances. In the initial evaluation of the patient, and in addition to the collection of a thorough medical, psychiatric and drug/toxic history, a complete physical and neurological examination should be completed, as well as the performing of laboratory tests and other investigations to enable a more accurate diagnosis and rule out the organic etiology. ${ }^{[4]}$ It is important to note that catatonia is associated with life-threatening medical complications (pulmonary embolism, pneumonia, myocardial infarction, dehydration, hyponatremia, renal failure, etc.) that must be recognized in time for early treatment. ${ }^{[4]}$

Once catatonia is recognized, the work plan will consist of the specific treatment of the syndrome, the underlying causes and possible complications. Benzodiazepines, especially lorazepam, are the drugs of choice for the acute management of catatonia. For patients unresponsive and who are in a serious clinical condition, electro-convulsive therapy (ECT) can be beneficial. We should start a lorazepam trial with the administration of 1-2 $\mathrm{mg}$ intramuscular or orally. After $3 \mathrm{~h}$, and if there is no response or if this is partial, we should repeat the dose. This same dose may be repeated after 3 hours. The next day will administer the total dose reached previously in one or more doses taking care not to sedate the patient. This dose will be administered on the following days until complete resolution of symptoms. An initial positive response usually predicts a more sustained response with further doses of treatment. The final effective dose of lorazepam that achieved a complete resolution of the catatonic signs will then be maintained for some days until the treatment of the cause of catatonia is well under way. A premature suspension of treatment with lorazepam is likely to provoke a recurrence of the catatonic symptoms..$^{[5]}$

In this issue of Journal of Neurosciences in Rural Practice, Muneer present a case of catatonia in a patient with bipolar depression who was treated with levetiracetam. ${ }^{[6]}$ The 
possible organic etiology was ruled out after obtaining normal results on physical examination and the laboratory tests requested. Because of exceptional situations described in the case, benzodiazepines or ECT were not used. The clinicians decided to use levetiracetam due to its action as a possible mood stabilizer and 6 weeks after the complete resolution of catatonic symptoms is achieved. This improvement would have occurred due to the mechanism of action of this anticonvulsant on GABAergic and glutamatergic neurotransmission whose dysfunction is implicated in the pathogenesis of this syndrome. ${ }^{[7]}$

This would be the first case of successful use of levetiracetam in catatonia in contrast to the report of a patient who developed this syndrome following the use of the same drug. ${ }^{[8]}$ Catatonia is a effectively treatable neuropsychiatric syndrome in which benzodiazepines and ECT have proven efficacy and safety. These will always be our first choices of treatment before the use of others drugs, like this anticonvulsant, that we could use in exceptional cases such as presented here.

\section{Walter Jaimes-Albornoz}

Arrasate-Mondragon Mental Health Center, Basque Health Service - Osakidetza, Basque Country, Spain

Address for correspondence: Dr. Walter Jaimes-Albornoz, Arrasate-Mondragon Mental Health Center, Basque Health Service - Osakidetza, Erguin 23, Bajo, CP 20500, Arrasate-Mondragon, Basque Country, Spain. E-mail: walter.jaimesalbornoz@osakidetza.net

\section{References}

1. Fink M. Rediscovering catatonia: The biography of a treatable syndrome. Acta Psychiatr Scand Suppl 2013;1-47.

2. Sienaert P, Rooseleer J, De Fruyt J. Measuring catatonia: A systematic review ofrating scales. J Affect Disord 2011;135:1-9.

3. Tandon R, Heckers S, Bustillo J, Barch DM, Gaebel W, Gur RE, et al. Catatonia in DSM-5.Schizophr Res 2013;150:26-30.

4. Jaimes-Albornoz W, Serra-Mestres J. Catatonia in the emergency department. Emerg Med J 2012;29:863-7.

5. Rosebush PI, Mazurek MF. Catatonia and its treatment. Schizophr Bull 2010;36:239-42.

6. Muneer A. Catatonia in a patient with bipolar disorder type I. JNeurosci Rural Pract 2014;5:314-6.

7. Northoff G. What catatonia can tell us about "top-down modulation": A neuropsychiatric hypothesis. Behav Brain Sci 2002;25:555-604.

8. Chouinard MJ, Nguyen DK, Clément JF, Bruneau MA. Catatonia induced by levetiracetam. Epilepsy Behav 2006;8:303-7.

\begin{tabular}{|l|l|}
\hline \multicolumn{2}{|c|}{ Access this article online } \\
\hline Quick Response Code: & \\
\hline & \\
\hline
\end{tabular}

